\title{
Living Voodoo Priest Huoshan Dendrobium Stem Cultivation Technology Research
}

\author{
Le Guiqi, Liu Ningfang, Zuo Jiachun \\ Nanchang Educational Science Research Lustitute 330006 \\ Jiangxi Science\&Technology Normal University
}

Keywords: Huoshan dendrobium stem; Biological characteristics; Pick cultivation techniques

\begin{abstract}
Greenhouse cultivated huoshan dendrobium stem, compared with wild huoshan dendrobium stem, has obvious difference whether towards its appearance and internal quality. Exploring experiment results on somaclone, planting seedlings, living voodoo priest engraftment technology, and outdoor cultivation huoshan dendrobium stem show that living voodoo priest cultivation huoshan dendrobium stem has stout stem, deep color, with the connotation of caulis dendrobii alkali and more rich caulis dendrobii polysaccharide, so it is favored by consumers.
\end{abstract}

\section{Introduction}

Caulis dendrobii in Dabie mountain area of Huoshan County in Anhui province in China are mainly huoshan dendrobium stem, copper caulis dendrobii and tin caulis dendrobii three kinds, and there is a small amount of Henan caulis dendrobii. The local people regard the 4 kinds of caulis dendrobii collectively as huoshan dendrobium stem. Huoshan dendrobium stem is one of LanKeShi herbaceous plants, which grows in the mist of cliffs or towering old trees, and the main components are caulis dendrobii alkali and caulis dendrobii polysaccharide. According to Chinese medical records, huoshan dendrobium stem is an amazing product, which nourishes Yin, benefits stomach, lung, stops cough and enhances immune activity, and so on. Wild dendrobium stem has low reproduction rate and slow growth, so its price is much higher than American ginseng processing products, and difficult to meet the needs of domestic and foreign markets and clinical medicine. Therefore, huoshan dendrobium stem's reproduction technology development is rapid, and artificial greenhouse cultivation is a success. But now people lack the knowledge of huoshan dendrobium stem's growth condition, the rule of material synthesis and metabolism of decomposition, and huoshan dendrobium stem research is not enough, so they are unable to create the best cultivation environment, which affects the quality and yield of artificial planting huoshan dendrobium stem. On the basis of research and summarization of the experiences of artificial planting huoshan dendrobium stem and reference, a large number of literatures, in combination with biological characteristics of huoshan dendrobium stem, the author explores the living voodoo priest huoshan dendrobium stem cultivation technique in order to provide reference and theoretical basis for largescale cultivation of high-quality huoshan dendrobium stem.

\section{Biological Characteristics of Huoshan Dendrobium Stem}

Morphological characteristics. Huoshan dendrobium stem, stem erect, succulent, $3 \sim 9 \mathrm{~cm}$ long, tapering upward from the base, the base coarse about $3 \sim 10 \mathrm{~mm}$, not branched, $3 \sim 7$ days with $3 \sim$ $8 \mathrm{~mm}$ internode length, yellowish green, sometimes with violet red spots, dry pale yellow. Leaves leathery, $2 \sim 3$ alternates in the upper portion of the stem, oblique, ligule oblong, $9 \sim 21 \mathrm{~mm}$ long, 5 $\sim 7 \mathrm{~mm}$ wide, apex obtuse and emarginated, base amplexicaul sheath; Leaf sheath membranous, persistent. Raceme $1 \sim 3$, from the old stems from the upper leaves with $1 \sim 2$ flower; Inflorescence handle $2 \sim 3 \mathrm{~mm}$ long, base is $1 \sim 2$ pieces of sheath; Sheath paper, ovoid, lanceolate, $3 \sim 4 \mathrm{~mm}$ long, apex sharp; Buds plain ribbon chestnut, ovate, $3 \sim 4 \mathrm{~mm}$ long, apex sharp; Pedicel and ovary yellow green, 2-2.7 cm long; Flowers yellowish green, conduct; Sepals lanceolate, oval in $12 \sim 14$ mm long, $4 \sim 5 \mathrm{~mm}$ wide, apex obtuse, article 5 of arteries and veins; Lateral sepals sickle lanceolate, $12 \sim 14 \mathrm{~mm}$ long, $5 \sim 7 \mathrm{~mm}$ wide, apex obtuse, base skew; Calyx nearly rectangular 
capsule, $5 \sim 7 \mathrm{~mm}$ long, terminal suborbicular; Petals oblong, oval usually $12 \sim 15 \mathrm{~mm}$ long, $6 \sim 7$ mm wide, apex obtuse, article 5 of arteries and veins; Lip nearly rhomboid, length and width is about equal, 1-1.5 cm, base cuneate and a corpus callosum, the upper slightly 3 crack, between the two side lobes densely short hair, near the base of dense grow white hairs; Lobes in a half-circle triangular, apex nearly a blunt tip, base densely grow white hairs and a yellow horizontal oval plaque; Columnpillar pale green, ca. $4 \mathrm{~mm}$ long, $7 \mathrm{~mm}$ long columnpillar foot; Columnpillar foot base yellow, thick grow white hair, on both sides of the accidental tooth; Medicine hat green white, almost half spherical, $1.5 \mathrm{~mm}$ long, apex slightly concave. Flower for five months.

\section{Growth characteristics.}

1. The growth environment. Huoshan dendrobium stem is not the evolution of aerial orchid, and its growth condition is extremely harsh. More on the stream valley cliff beside river waters and ditches, often with moss, herba pyrrosiae plant volatile oil. It likes cool, moist, and ventilation foggy microclimate. It begins to grow in the temperature of $14{ }^{\circ} \mathrm{C}$, prefers temperature of $20 \sim$ $26{ }^{\circ} \mathrm{C}$ and more than $80 \%$ humidity air; when temperature is above $30{ }^{\circ} \mathrm{C}$, with relative humidity under $20 \%$, the veins become yellow, with drooping leaves and stems are shrinking; too many cliff seam water, seepage roots are easy to rot to death.

2. Growth cycle. Huoshan dendrobium stem is of pseudobulb points nie ability, and under natural condition, it sprouts slow, usually 1 year only 1 stalk, points nie stem growth also increases every year, so we can generally determine the strain plexus "age" according to the number of stem and stem height. Annual new stem initiates fibrous root, and spring and summer are the growth peak, autumn leaves do not fall off and enter a dormant state, so it belongs to the evergreen life forms; Biennial stems mainly accumulate nutrition and fertile, and generally no longer grow. After the second growing season, leaves on stem gradually fall off; Born three years flowering stems bloom and bear fruit, the stem leaves no longer grow after initiation of new leaves; Four years stem loses points nie ability; Five years born and six years born stem wilt and die.

3. The phenophase. According to the growth of flower bud, bud development and tepals degree of huoshan dendrobium stem, the single flowers blooming is divided into five stages, namely, bud, early budding stage, flowering stage, full bloom and wither; Each year in Huoshan county in Anhui province nature reserve in mid-May, huoshan dendrobium stem germinates minority single flower flower buds, and the majority is still inflorescence package; buds begin to bloom in early June, which is in full bloom since the middle of June; spend amount is sharply reduced at the end of June; early July is the end of flowering. Huoshan dendrobium stem has plant flowering in early June to early July, but the flowering period mainly concentrates in late June, and the time from flower bud germination to flowering time is about 35 days.

\section{The Somaclone of Huoshan Dendrobium Stem}

Based on biological characteristics of huoshan dendrobium stem, combining the successful experience of artificial planting huoshan dendrobium stem, assume that living voodoo priest cultivation huoshan dendrobium stem experiences: somaclone, cultivation and living pick three stages:

Somaclone training path.Currently, caulis dendrobii organization has seeds, protocorm, artificial seeds, and no vaccine seedling stem section, etc. for explant. Regeneration plant getting ways mainly have the following kinds: one is seed-original bulb- small plants; The second is the seed- original bulb-callus- clump buds-rooting seedlings; Three is seed - protocorm -no vaccine stem segment - small plants; Four is seed - callus- protocorm - small plants; Five is the original bulb - artificial seeds-seedlings; Six is the stem tip - callus - clump buds- rooting seedlings, etc. We adopted in the experiment sow the seed - protocorm - small plant, and get the somaclone greenhouse cultivation in 1 year.

Tissue culture matrix.In earth bottle's basic medium, add a certain concentration of activated carbon, and reagents such as NAA and MS, which is advantageous to the somaclone taking root, promotes the growth of the somaclone, and enhances the survival rate of transplant. To join in the 
basic medium potato juice or banana juice, mungbean sprout juice, apple juice and so on also has the function of promoting growth and root growth of somaclone. Experiments show that banana juice boost is the most significant.

\section{Huoshan Dendrobium Stem Planting Seedlings}

Somaclone needs 1 year transplanting greenhouse cultivation, and when somaclone transplanting, it is subject to various factors influence such as quality, cultivation matrix, light, temperature and humidity. To make huoshan dendrobium stem somaclone adapts to the cultivation of greenhouse environment as soon as possible, we should do the following work well.

\section{Melting plants and out of the bottle.}

1. The seedlings. Move the somaclone to smelting plants room for $2 \sim 3$ weeks for smelting plants, let the somaclone gradually adapts to the natural environment, and get it out of bottle when the leaves color is thick and grows strong.

2. The container seedlings. Multiplication algebraic within 10 generations, plants within $3 \mathrm{~cm}$ long, fleshy stems have $3 \sim 4$ internode with $4 \sim 5$ leaves, the leaf color is normal; Root length is more than $3 \mathrm{~cm}$, with $4 \sim 5$ roots, and root is white with green with no black root, no abnormalities, without variation.

3. Out of the bottle. Remove medium gently together with seedlings, eliminate pollution seedlings, little naked root or roots. Robust somaclone should be washed with water, particularly wash out AGAR, lest cause roots rotted AGAR mould, and then use clean water rinse, dry it for the preparation of transplanting; after cleaning, soak the root of bare root or less somaclone in 100 $\mathrm{mg} / \mathrm{L} \mathrm{ABT} \mathrm{ABT} \mathrm{for} 15 \mathrm{~min}$ to induce their rooting; for pollution seedlings, after cleaning, use carbendazim 1000 times to immerse the plant seedlings for $10 \mathrm{~min}$, and transplant, manage them in a separate area.

\section{Greenhouse cultivation and cultivation matrix.}

1. Greenhouse cultivation. According to the biological characteristics of huoshan dendrobium stem, fully considering the field of illumination, temperature and humidity, ventilation and other natural factors (typically choose the distribution of wild dendrobium stem area). Build greenhouse cultivation as far as possible to meet the best nutritional requirements of huoshan dendrobium stem growth, greenhouse cultivation should satisfy the following conditions:

(1) The greenhouse size should be designed reasonably according to the size of the planting scale; generally, the greenhouses are around $30 \mathrm{~m}$ long and $6 \mathrm{~m}$ wide, with shoulder height $1.8 \mathrm{~m}$, and the total $4 \mathrm{~m}$ high; between planting seedling beds, reserve $1 \mathrm{~m}$ or so wide roads for convenient management. Greenhouse building can choose steel skeleton greenhouses, which is durable, but one-time investment cost is higher; Also it can choose concrete structures, so a one-time investment cost is low, but life is short, after years, the total cost is no lower than the former. Roof covering plastic is shade nets with plastic film and 70\%shade degree. Insect nets are fitted around the greenhouses and entry. Cultivation greenhouse needs water, electricity, pathways, and best in relative installation of automatic or manual control spray system (spray, spray fertilizer, spraying);

(2) Build elevated planting seedling bed to make it easily to control the moisture, open air, so as to provide favorable conditions for the growth of somaclone. Angle steel, wood and other materials are seedbed framework, and then lay about $5 \mathrm{~mm}$ aperture solid plastic flat as cultivation matrix bearing surface. Seedbed is $1.2 \mathrm{~m}$ wide, with overhead height of $40 \mathrm{~cm}$.

2. The cultivation matrix. Different cultivation matrix directly affects huoshan dendrobium stem transplanting survival rate, growth, reproduction, and production. The experimental results show that when limestone gravel filter layer is $2 \mathrm{~cm}+5 \mathrm{~cm}$ ground bark are matrix, huoshan dendrobium stem planting seedling grows best. Bark is loose and breathable, and can keep moisture and fertilizer, which is suitable for the requirements of root growth. Limestone particles with a suitable amount of sawdust or pure limestone particles can be better matrix of caulis dendrobii planting seedlings. 


\section{Cultivation management and prevention and cure of diseases and pests.}

The study found that spring March is the best time for transplanting huoshan dendrobium stem somaclone to survive, and cultivation of seedlings growing is closely related to the size of the somaclone. The smaller grown somaclone, due to less accumulation of organic matter and energy, is of weak ability to adapt to the outside world and resistance, so it grows slowly.

1. Light, temperature and humidity. Relevant research thinks that huoshan dendrobium stem planting seedlings are appropriate in cool, moist, and open air to grow, and the optimum temperature for the growth is $20 \sim 30{ }^{\circ} \mathrm{C}$. At high temperature in summer, cultivation greenhouse must have ventilation cooling, at the same time, carry on spray cooling moisture; Winter low temperature can be controlled by heating around $10{ }^{\circ} \mathrm{C}$. Humidity of the air within 1 week after transplanting should be kept at around 90\%; after 1 week, air humidity can be kept at 70\% 80\%. Light intensity should be controlled within $30000 \mathrm{~lx}$, such an environment is most suitable for cultivation of seedling growth.

2. Hormones, nutrition and moisture (1) Nutrition. Relevant studies suggest that after somaclone was cast transplant $15 \mathrm{~d}$, half times nutrient solution is the best. Study found huoshan dendrobium stem application of nutrient solution (mainly composed of potassium nitrate, ammonium sulfate, magnesium sulfate, ferric sulfate and potassium dihydrogen phosphate and so on, proper nutrient solution concentration can greatly improve the output of huoshan dendrobium stem. Huoshan dendrobium stem commonly uses foliar fertilizer, $(\mathrm{N}, \mathrm{P} 2 \mathrm{O} 5, \mathrm{~K} 2 \mathrm{O}=25: 13: 13$, the total $\mathrm{N}, \mathrm{P}, \mathrm{K}$ content is $51 \%$, trace element $\mathrm{B}, \mathrm{Fe}$, zinc, $\mathrm{Cu}, \mathrm{Mn}$, Mo content is $1 \%$ ) can promote the growth of huoshan dendrobium stem elongation, internodes, longer bold diameter, fresh weight per plant, on average, is significantly higher than the control. (2) Hormones. Relevant studies suggest that application of exogenous auxin (such as GA, 6 - BA) promotes obvious production effect; the experimental results show that hormone treatment can enhance the activity of active oxygen scavenger, reduce the MDA from membrane lipid peroxidation, help improve the resistance of huoshan dendrobium stem. (3) Moisture. Just transplanted somaclone is the most sensitive to water; water should not be too high or too low. Large area of cultivation askes for substrate wetting, but not water, keeps more than $80 \%$ relative humidity of the air. Best use water sprinkler irrigation, and not be rushed. Different seasons have different water quantity, summer means high temperature and evaporation is big, basically water every day; winter means low temperature, and moisture is not easily to lose, therefore need to depend on water content, if the cultivation matrix is partial dry, supply some water.

3. Cultivation seedling diseases. Huoshan dendrobium stem planting seedling diseases basically are soft rot, black rot, anthrax, etc. Soft rot disease is quick and when serious, the plants are wet rot decay and collapsed; Melasma harm blade blastes the blades; Caulis dendrobii anthracnose leaves and fleshy stems, and suffered blades are in black or with brown disease spots. According to these common diseases, we should strengthen the cultivation of greenhouse management, pay attention to the ventilation pervious to light, reduce relative humidity appropriate, and timely deliver mechanism and strain from pull out.

4. Planting seedling pests. Huoshan dendrobium stem planting seedlings are mainly pests' snail, fe shield pest, and starscream, etc. Among them, snail is a common pest, which harms young stem, leaves, buds and young fruit, and is vailable for ensnared, artificial hunting or sprinkled lime, ChaFu prevention; Caulis dendrobii fe shield pest eat juice at the back of plant leaf edge. Burn old branch concentrated with shield pest or to kill them with $40 \%$ dimethoate ec 1000 times spay; Starscream can remove weeds from the environment, or spray mites agent $800 \sim 1000$ times to lower poison prevention and control. Huoshan dendrobium stem is precious medicinal material, therefore, huoshan dendrobium stem plant diseases and insect pests prevention and control should be prevented as much as possible, physical prevention and control and biological control should be given priority to, or use low-toxic pesticide; Once found plant diseases and insect pests, corresponding measures should be taken in time to avoid the deterioration of the plant diseases and insect pests affection. 


\section{Living Voodoo Priest Cultivation}

\section{Transplanting time and method.}

1. Transplanting time. Huoshan dendrobium stem live pick plant is appropriate in spring in March or fall in September. When the temperature is too low or too high, it is unfavorable for pick cultivation. Experimental results show that spring is more suitable for living accessory plant huoshan dendrobium stem, because in March, the climate goes up with bright sunshine and gentle breeze, natural temperature, sunshine, rain conditions, such as highly caulis dendrobii stem base of axillary bud germination, grow for germ aerial roots to absorb nutrients and moisture,and achieve the growth of roots and shoots after first. Autumn engraftment enjoys natural climate with root growth, but the roots quality, quantity and speed are less than the spring.

2. The transplanting method. In mountain forest, the choice of thick tree stem, more water, exuberant canopy, loose bark, and longitudinal fissure evergreen tree (such as yellow barrels, Chinese sapium, persimmon, tung, green bar, camphor, nanmu, Chinese beech trees, etc.), in a relatively flat, thick trunk or branches of sunken place or every $30 \sim 50 \mathrm{~cm}$ gap, cut a shallow cut with knife, and strip off some bark, and then fixed to the segments of the tree with prepared rope or woven mesh belt around caulis dendrobii seedlings roots, then put cow dung slurry (with cow dung and mud mix) around the roots and bark in the ditch. To prevent planting seedlings from wind and rain damage, in general, bind caulis dendrobii seedlings roots with the epiphytes tree to facilitate its new root length, and the close up the growth of trees. On the tree planting, it should from the topdown, and the lowest place should be above 1 meter.

\section{Daily management and collection.}

1. Daily management.

2. Caulis dendrobii harvest. Living voodoo priest planting huoshan dendrobium stem can harvest in $1 \sim 2$ years, and the optimum stage for harvest is after "the beginning of winter" to "Qingming festival", when caulis dendrobii has stopped growing at this time with solid, full and dry bark. To increase the production of huoshan dendrobium stem, and ensure the good quality, generally we should adopt "two for one", namely the harvest for 2 years and over huoshan dendrobium stem, and leave the less than 2 years huoshan dendrobium stem for reproduction. After harvesting, we should pay attention to timely spraying protective fungicide to prevent the happening of the disease.

\section{Summary}

Huoshan dendrobium stem somaclone, after 1 year of greenhouse cultivation, is out of the bottle to greenhouse cultivation, and after cultivation of 1 year again, forms the cultivation of seedlings. Each spring, plant robust seedlings engraftment in appropriate environment, half sunshine trees covered with moss. In nature, on its own aerial root, absorb sunshine, rain and dew of nature to grow. The year of engraftment, after the beginning of winter, can harvest huoshan dendrobium stem, and the second year is for living voodoo priest caulis dendrobii in season.

Living voodoo priest cultivation huoshan dendrobium stem, compared with artificial greenhouse cultivation huoshan dendrobium stem, its cultivation time, cultivation techniques and cultivation of costs are almost equal, but the harvesting caulis dendrobii quality from the two types of cultivation is obviously different. Living voodoo priest cultivation huoshan dendrobium stem absorbs the essence of nature. Looking from the exterior, caulis dendrobii stem is stout with deep color and its connotation of caulis dendrobii alkali and caulis dendrobii polysaccharides are richer. Living voodoo priest cultivation of huoshan dendrobium stem effect is more apparent, and so it is favored by consumers.

\section{References}

[1] Wu Huqi, Luo Jianping. The research progresses of huoshan dendrobium stem [J]. Shizhen National Physician and National Medicine, 2010, (1) : 0208-04. 
[2] Wei Fengjuan. Iron caulis dendrobii tissue cultivation and cultivation technology research progress [J]. Journal of Guangdong Agricultural Science, 2010, (4) : 0081-05.

[3] Cai Yongping, Li Ling, Li Hesheng, etc. Three kinds of dendrobium stem leaf photosynthetic characteristics and its response to light in Huoshan County [J]. Chinese Herbal Medicine, 2005, 4 (4) : 0586-05.

[4] Li Miao, Wang Lin, Chen Qun, etc. Huoshan dendrobium stem tissue cultivation and rapid reproductive cloning technology [J]. Journal of Biotechnology Drugs, 2011, 19 (1) : 011-015.

[5] Ni Qinwu, Chen Lianqing. Zhejiang Fuyang grass wild black section morphology research [J]. Journal of Zhejiang Institute of Traditional Chinese Medicine, 2004 (3) : 59-60.

[6] Xie Yinfeng, Xu Li, Zhang Zhimin, etc. Several kinds of organic additives effects on huoshan dendrobium stem tube seedlings physiological characteristics [J]. Journal of Northwest Forestry College, 2011, 26 (1) : 77-87.

[7] Xing Fusang, Feng Jindong, Liu Lingfeng, etc. The general research situation of tin caulis dendrobii cultivation technique [J]. Shizhen National Physician and National Medicine, 2010, 13 (9) : 0559-01. 